\title{
Typologie d'erreurs lexicales : difficultés et enjeux
}

\author{
Caroline Masseron \& Olha Luste-Chaa \\ Université Paul-Verlaine de Metz \\ masseronj@,wanadoo.fr \\ olgaluste2@yahoo.fr
}

La typologie des erreurs en orthographe, telle qu'elle a été conçue par N. Catach et son équipe (Gruaz et Catach 1979, Catach 1980), a connu immédiatement un grand succès, si l'on en juge par les applications auxquelles elle a donné lieu en didactique de l'orthographe (Gey 1979, Honvault 1985, Cogis 2005). En effet, d'une part, cette typologie a été perçue comme permettant un affinement sensible - fondé linguistiquement - de la description des erreurs orthographiques ; d'autre part, son apparition a coïncidé avec une période de réflexion très productive sur l'erreur (Reuter 1984) et l'évaluation (Groupe EVA 1991), et ce notamment en contexte de production d'écrits (Halté 1984, Garcia-Debanc 1990), domaine qui laissait traditionnellement les maîtres très démunis devant les infractions du code et les maladresses d'expression ${ }^{1}$. Mais par-dessus tout, sans doute faut-il voir dans l'explication de ce succès immédiat la convergence entre un domaine dont l'enseignement est problématique et une description homogène dont la mise en cohérence théorique et didactique est affichée comme un objectif central; la langue y est décrite comme un plurisystème dont la complexité et les interférences de ses réglages engendrent par nature, lors de l'apprentissage de l'écrit, des erreurs d'encodage, lesquelles erreurs sont analysées et répertoriées par la typologie construite à cet effet. Outre la cohérence de la description, cette typologie présente enfin un avantage crucial qui est celui de son exhaustivité supposée. Tous les types d'erreurs y sont représentés, codés sur la base de descripteurs graphiques, configurant un tableau synthétique et assez commode. La réalisation de cette typologie d'erreurs en orthographe est facilitée par l'existence et la permanence des réglages formels et univoques du code écrit, quoi qu'il en soit des zones très marginales de tolérance orthographique.

On s'en doute déjà, rien de tel avec le lexique qui n'offre pas les mêmes « facilités » que l'orthographe. L'idée même d'erreur lexicale pose problème à l'interprétant. Deux cas bien connus illustreront cette difficulté, le cas des erreurs non marquées ou indirectes et celui du « mal dit » à caractère grammatical ou stylistique. L'erreur peut être indirecte, tout d'abord, quand elle procède d'une sorte de calcul d'inférence, sur la base de l'absence - jugée fautive - d'une unité lexicale à la surface du texte. Ou bien le jugement d'erreur est discutable et sera discuté par deux correcteurs distincts, par exemple si le jugement d'enfreinte lexicale recourt à une norme, implique un jugement de valeur ou de conformité à un registre de discours ou un genre d'écrit. Enfin, dernier facteur aggravant : par différence avec ce qui s'observe en orthographe, les erreurs lexicales se manifestent en réception et en production de textes ${ }^{2}$, selon des voies très probablement distinctes. Faut-il dans ces conditions maintenir le cap annoncé en s'obstinant à vouloir dresser une typologie des erreurs lexicales?

Les arguments susceptibles d'étayer un tel projet tiennent principalement au statut du lexique en langue et en discours. Sur un versant, celui du lexique en puissance, nous trouvons l'organisation du lexique et la construction de ses unités, sa description en langue et sa disponibilité ou son accessibilité, toute théorique, chez un locuteur quelconque ; sur l'autre versant, celui du lexique en usage, se retrouvent des emplois circonstanciés et fonctionnels, actualisés et déterminés à la fois par l'identité de l'usager et par l'objet de discours à traiter. Une telle partition, pour schématique qu'elle soit, laisse entrevoir que les occurrences lexicales d'un discours sont à l'interface de l'intention signifiante au sens le plus large et de la réalisation singulière du discours. C'est pourquoi on considèrera que les choix lexicaux, ici et pour commencer en production d'écrit, constituent un indicateur de premier ordre pour interpréter dans toute leur complexité les performances scripturales d'un apprenant. Par conséquent, en nous inspirant des typologies d'erreurs en orthographe mais sans toutefois sous-estimer les problèmes qu'elles ont parfois contribué à masquer, nous souhaitons ébaucher une problématique de l'erreur lexicale en situation (scolaire) de production 
d'écrit. L'article s'attachera surtout à cerner les contours épistémologiques de la démarche générale et à en illustrer quelques aspects singuliers. Étant donné la finalité didactique du projet, notre propos sera sous-tendu par la question des besoins langagiers, des aides à l'acquisition lexicale et bien sûr par celle, difficile entre toutes, de l'enseignement du lexique. Dans cette perspective, nous entendons par erreur la manifestation d'un défaut de maîtrise langagière, identifiée par le biais d'une unité, simple ou complexe, qui s'apparente au stock lexical de la langue. L'unité lexicale est en quelque sorte vecteur langagier, que le lexique soit convoqué sur son versant constructionnel (en puissance) ou pragmatique (en usage).

\section{Problèmes soulevés par l'établissement d'une typologie d'erreurs lexicales : localisation, identification et dénomination de l'erreur}

Pour constituer un répertoire d'erreurs lexicales, il faut préalablement qu'on ait pu tout d'abord attester les erreurs en question dans un corpus ou un échantillon de corpus, pour en éprouver la représentativité en termes quantitatifs (fréquence, nombre de locuteurs) et qualitatifs (au centre ou à la marge des questions lexicales; degré de gravité de l'erreur rencontrée; productivité de l'infraction et du domaine de correction). Une telle assertion est moins triviale qu'elle ne paraît. Deux options se présentent en effet et non une seule.

\subsection{Quelle classification pour quel corpus de textes ?}

La première option consiste en une sorte de relevé aléatoire. Sont consignées alors toutes les erreurs d'où qu'elles proviennent. On peut même envisager d'élargir les données aux corpus non scolaires, la presse par exemple. Cette solution nous oblige alors à envisager un classement qui demeure quasi-inhérent au lexique lui-même, c'est-à-dire, pour une part au moins, largement indépendant des usages (les énoncés et leurs auteurs) et des contextes qui ont actualisé l'erreur relevée. Par ailleurs, les critères de classification doivent être, d'emblée, opératoires, et dès lors préexister : leur formulation doit avoir précédé le relevé qui n'est plus, dans cette hypothèse, qu'une simple illustration des jugements de disqualification.

La seconde option consiste à privilégier des corpus à peu près homogènes. Nous entendons par là des ensembles de textes qui traitent d'un même thème et dont les producteurs sont, dans un premier temps au moins, proches d'un point de vue socio-scolaire. On peut par exemple constituer en corpus un ensemble de copies qui ont répondu à la même consigne. Cette option présente l'avantage d'un vocabulaire à peu près prédictible, y compris du point de vue de l'environnement syntaxique des occurrences lexicales.

L'alternative que nous venons d'évoquer - relevé aléatoire dans un corpus vaste et hétérogène et classification préexistante, ou corpus homogène de moindre ampleur et analyse de l'erreur lexicale en contexte qui détermine une indentification provisoire par familles de problèmes - permet au moins à titre transitoire d'éclaircir notre démarche. Nous retenons l'option d'un corpus restreint et d'une analyse qualitative comme un premier stade nécessaire pour appréhender l'unité lexicale dans ses interrelations avec les dimensions (micro- et macro-) sémantiques et syntaxiques.

Précisons enfin que le corpus restreint auquel nous consacrons cette étude est un corpus FLE; nous en donnerons un peu plus loin les conditions de recueil. C'est lui, en tout cas, qui alimente principalement le classement d'erreurs lexicales que nous ébauchons. Mais nous escomptons de cette première étape tout empirique qu'elle nous aide à mieux concevoir le rôle structurant que joue le lexique dans la maîtrise écrite du français, que le scripteur soit ou ne soit pas natif de cette langue. Au-delà en effet de cette première étape, nous entendons nous ateler pour la question lexicale, dans les deux contextes FLM et FLE, à la conception de seuils et d'objets d'acquisition.

\subsection{Erreurs lexicales en contexte FLE : enjeux d'un recueil spécifique}

En didactique des langues étrangères (désormais DLE) et notamment en didactique du français langue étrangère (DFLE), dont relève le travail que nous présentons, la notion d'erreur et celle d'erreur lexicale en particulier font l'objet de réflexions et d'analyses depuis une trentaine d'années (Corder 1980, Perdue 
1980, Porquier et Frauenfelder 1980, Porquier 1977 et 1990, Doca 1981, Les Langues Modernes 1987, Favre 1995). Étant donné la quantité d'éléments linguistiques à traiter et la complexité de leurs interelations, on sait bien que l'apprentissage d'une langue comporte - presque par définition - des approximations qui sont la trace des essais et des erreurs successifs dans la langue visée ; le code de celleci présente de fait des «obstacles » que le locuteur doit traiter de façon quasi-simultanée. L'unité lexicale joue, dans cette perspective, le rôle d'un indicateur pour la compréhension du sous-système fonctionnel qu'elle actualise.

Conscients donc de la réalité que tout apprenant en langue étrangère (LE) commet des erreurs lors de son apprentissage, nombre de didacticiens et de chercheurs en acquisition de LE adoptent un point de vue constructiviste au sujet de traitement et d'analyse des erreurs (Tréville et Duquette 1996, Véronique 2000, Vigner 2001, Veltcheff \& Hilton 2003). En effet, en FLE ainsi qu'en français langue maternelle (FLM), les erreurs commises par les apprenants se révèlent comme « porteuses du sens » (Tarin 2006) autant pour l'enseignant que pour l'apprenant.

Pour l'apprenant, la prise de conscience de l'erreur commise, l'explication et la compréhension de sa nature favorisent la progression dans l'apprentissage, en enrichissant par ailleurs les réseaux sémantiques de son lexique mental. Sont en jeu la perception et l'analyse des formes-sens réalisées, par différence avec les formes-sens qui sont attendues.

Pour le formateur, les erreurs lexicales jouent, nous l'avons dit, un rôle d'indicateur du niveau de langue atteint par l'apprenant et sont susceptibles de mettre en évidence à la fois les ressources linguistiques dont disposent les apprenants et en même temps les besoins lexicaux et plus généralement linguistiques, qu'il convient de l'aider à construire. Les recherches en didactique ont montré que, pour qu'une correction soit recevable, elle ne devait pas se limiter à la simple «correction» de la production erronée, stratégie insuffisante si l'on veut étoffer l'acquisition de tours nouveaux et conscientiser une démarche - offensive mais coûteuse sans doute en erreurs commises - qu'on pourrait qualifier d'essais langagiers successifs. Dès lors, le type, la fréquence de certaines erreurs et le niveau auquel appartiennent les erreurs identifiées doivent pouvoir, positivement, fonder une évaluation diagnostique.

L'intervention de l'enseignant requiert un cadre explicatif qui soit à la fois ouvert et rigoureux, où il convient de mener une réflexion approfondie et multifactorielle sur les sources de l'erreur recensée. Rappelons pour mémoire les facteurs d'erreurs déjà inventoriées par la recherche en didactique du FLE ; elles sont ici formulées dans des termes qui demeurent généraux, qu'ils soient acquisitionnels ou contrastifs :

- Les influences des acquis antérieurs, en particulier de la langue maternelle et éventuellement d'autres langues étrangères apprises avant le français, le point de vue qui s'inscrit dans l'analyse contrastive ;

- Les stades du développement de l'interlangue de l'apprenant qui présenterait un système intermédiaire évolutif dans l'acquisition d'une LE entre la langue maternelle et la langue cible (Klein 1989, Bogaards 1994, Vogel 1995, Tréville et Duquette 1996);

- Les stratégies d'apprentissage adoptées par l'apprenant.

Il nous semble nécessaire d'y ajouter le point de vue que nous défendons aujourd'hui et qui est, à nos yeux, important, à savoir les caractéristiques intrinsèques (et fonctionnelles) du système lexical de la langue cible, le lexique du français dans notre cas. Cet aspect est évidemment d'autant moins négligeable que les enseignants qui ne partagent pas - ou pas souvent - la langue maternelle des apprenants, ou que les groupes d'étudiants étrangers sont loin d'être toujours homogènes. Par conséquent, il est difficile de tenir compte de l'évolution de l'interlangue de chaque apprenant.

Nous voudrions à terme, au sujet des erreurs lexicales, contribuer à la discussion des similitudes entre les processus d'acquisition LM et LE (Bogaards 1994). Et ce pour les raisons déjà mentionnées du lexique considéré comme un analyseur fonctionnel d'erreurs identifiées et spécifiques, caractéristiques d'une production allophone ou francophone. 
Nos observations et analyses sont fondées sur le corpus de 8 textes produits par des étudiants - jeunes adultes - d'origine chinoise qui ont appris l'anglais en Chine comme première langue étrangère. Avant la production des textes qui nous occupent, ces apprenants ont bénéficié de 300 à 400 heures d'enseignement du français en Chine et ensuite, d'environ 300 heures de cours de français intensifs dans une université française.

\subsection{D’une stratégie de correction empirique à une démarche contrôlée qui s'appuie sur les indicateurs lexicaux}

Certains genres de l'écrit ou de l'oral - en particulier ceux qui ne relèvent pas directement d'une situation d'apprentissage - présentent des emplois d'unités lexicales qui, s'ils sont ressentis dans l'instant comme approximatifs ou fautifs, obéissent à un principe communicationnel d'économie : ils ne sont pas passibles d'une séquence d'enseignement. On citera comme exemple le courrier électronique dont l'usage, fréquent et rapide, est susceptible d'engendrer des équivoques fondées sur des erreurs de tout type. L'écrit cependant - y compris celui du courrier électronique - conserve la trace des erreurs commises. C'est sans doute l'une des raisons majeures pour lesquelles l'erreur en contexte écrit et plus précisément l'erreur lexicale s'analyse doublement : elle est objet de jugement normatif et elle renvoie à un usage fonctionnel du système linguistique (Frei 1929).

Les situations d'enseignement et d'apprentissage de la langue écrite accentuent évidemment le caractère normatif des jugements d'erreur. Le maître a intériorisé, étant donné son rôle de formateur, la finalité modélisante des situations d'écriture et des formes d'écrits et il s'est forgé une représentation globalement lacunaire ou défectologique des pratiques langagières de son public et des besoins de «correction » que ces pratiques traduisent. Le modèle «contre » la production d'erreurs, telle est en raccourci la situation d'enseignement de la langue et des textes, envisagée dans les représentations spontanées comme une situation correctrice. Ce point de vue nous paraît être un angle d'analyse tout à fait essentiel - en particulier, qu'il convient de ne pas refouler au profit d'une vision, pour le coup naïve et trop optimiste, de l'inventivité naturelle et à encourager chez l'apprenant -, dès lors que l'on envisage l'enseignement de la langue dans sa triple partition maître / apprenant / langue (lexique).

Compte tenu de ce qui vient d'être rappelé sur l'importance des tâches de correction dans le travail d'un maître dont l'enseignement porte sur la langue (FLM ou FLE) et les textes, l'analyse des erreurs lexicales, telle que nous la concevons ici, doit être élaborée à partir de cet acte correcteur du maître. Il s'agit en quelque sorte de renforcer chez le maître un point de vue linguistique et fonctionnel sur les erreurs lexicales qu'il relève lors de ses corrections.

Reprenons l'alternative évoquée précédemment pour caractériser deux familles de corpus (un corpus ouvert, qui tend à la banque de données, et un corpus restreint, plus fermé et homogène) entre un relevé aléatoire et un relevé prédéfini par un certain type de vocabulaire. Le premier relevé s'accompagne, en situation scolaire, d'une annotation standard des copies (Halté 1984). Il procède ponctuellement à des corrections spontanées, où le lexique occupe le même rang que d'autres domaines d'enseignement, la syntaxe, l'orthographe, la conjugaison. Les erreurs lexicales identifiées sont d'ordre constructionnel et procèdent d'une correction formelle par commutation stricte. Un exemple nous aidera à caractériser cette première branche de l'alternative. L'extrait qui suit est écrit par un élève francophone d'une quinzaine d'années qui a produit un écrit d'invention narrative à partir du roman d'Orwell, La ferme des animaux :

(...) puis il fallut voter, mais c'était connu d'avance, le « oui » le remportait haut la main (47 votes contre 1, celui de Napoléon) et on entendait par un cri uniforme : vive Boule de Neige. (2006)

Le professeur qui corrige cette copie souligne uniforme pour y sanctionner une erreur lexicale. On peut penser que le raisonnement du correcteur fait l'hypothèse d'une confusion paronymique entre unanime et uniforme. Ce qui n'est d'ailleurs guère contestable. Néanmoins, on peut regretter que l'erreur commise sur l'emploi de la préposition par (on entendait par un cri uniforme) n'ait pas été relevée (en l'espèce, soulignée $)^{3}$. On peut en effet penser que le choix de par n'est pas anodin et traduit à la surface du texte 
un lien de causalité entre l'uniformité du cri (effet) et son motif (à l'unanimité des votants, nombreux). Par s'interprète alors comme signifiant d'après ou si l'on en juge par (le cri des votants). Dans cette mesure, ce sont deux unités - par et uniforme - qu'il convient de souligner, et non une seule. Le seul soulignement, sous une rubrique étroitement lexicale, de l'unité adjectivale, uniforme, fait perdre la trace du lien sémantique entre les deux adjectifs uniforme et unanime, alors même que ce lien est réalisé (par) dans l'environnement de l'adjectif jugé fautif. La paronymie identifiée permet, enfin, de discuter l'erreur : y a-t-il une faute avérée dans le choix, en contexte, de l'adjectif uniforme ? Pour répondre, on reformulera l'énoncé en un tour qui autoriserait le maintien de l'adjectif ; une telle réécriture, figurée, pourrait être :

\section{(...) puis il fallut voter, mais c'était connu d'avance, le « oui » le remportait haut la main (47 votes contre 1, celui de Napoléon), ce qui se vérifia au cri uniforme poussé par les animaux : vive Boule de Neige.}

Cet extrait et la réécriture à laquelle nous l'avons soumise nous aident à formuler les trois hypothèses de travail suivantes :

- Le lexique n'est pas un domaine de compétence autonome et la commutation de formes n'est que l'une des opérations que peut prendre la «correction» lexicale. À cet égard, on ajoutera que le « correcteur » doit se départir (momentanément) de ses tâches de stricte correction (en langue) pour leur associer une lecture active au cours de laquelle il devient l'interprétant d'un discours complexe. Cette posture interprétative vaut essentiellement pour les interrelations entre langue et discours, à travers les erreurs lexicales manifestées.

- L'erreur lexicale, nous l'avons dit, est rarement passible d'un jugement simple ou univoque en juste ou faux: elle engage au moins une unité de l'environnement verbal. Les dimensions sémantique et syntaxique sont les premières, naturellement engagées par une erreur lexicale. Audelà, d'autres plans de structuration, comme l'énonciation ou le point de vue par exemple, sont concernés.

- L'unité lexicale, en tant que mot, ne constituerait-elle pas finalement une sorte d'artefact (F. Rastier 2007, communication orale), requis à la fois par le découpage de la chaîne écrite, par la déclinaison morphologique des catégories et par la série paradigmatique mise en nomenclature par le lexicographe? Qoui qu'il en soit, ces dimensions (la réalité lexicale en tant qu'unité perçue) sont prégnantes dans les représentations spontanées de tout un chacun, et il importe de ne pas les traiter légèrement.

- Dans une double perspective de recherche-action et de formation des maitres, il convient d'assortir les jugements d'erreurs d'une analyse et d'une réécriture du fragment erroné M. Monte et alii, 1992 ; C. Masseron 2001 et 2005). Ces pratiques sollicitent le « correcteur » pour qu'il verbalise - directement ou indirectement - son jugement d'erreur (norme, critère).

- Les réécritures pproposées, par leur nature même (la complexité du processus rédactionnel), mais du fait aussi de leur visée d'apprentissage, sont envisagées sur un mode scalaire ou gradué. Plusieurs variantes du segment erroné sont livrées concurremment, à des fins de comparaison.

Ces hypothèses, telles que nous les avons émises, ne doivent pas prêter à confusion : elles sont à l'usage exclusif des formateurs. En aucun cas, il ne faut y voir de préconisation pour un travail dans les classes. Nous souhaitons simplement orienter l'observation des formateurs responsables et pour cela, à titre temporaire, modifier les tâches de correction lexicale en une pratique conjointe d'écriture et d'analyse. Au centre de cette démarche empirique, réside la difficulté de s'accorder sur la «nature » de l'erreur analysée. D'autre part, la démarche contraint le correcteur à sortir des classifications traditionnelles en parties du discours ou en domaines d'enseignement. Dans ces conditions, répétons-le, le lexique a statut de vecteur langagier, et le désaccord, de levier pour une analyse plurifactorielle. 


\section{Classement des erreurs : première ébauche}

Notre première section a essentiellement posé les questions d'ordre épistémologique soulevées par la notion d'erreur lexicale : le corpus, le contexte scolaire et le protocole (professionnel) de correction ont ainsi été évoqués. Nous voudrions maintenant prolonger ces réflexions générales par une application qui illustrera en quoi une stratégie de réécriture contrôlée, un corpus limité et un relevé méthodique des erreurs permettent d'étoffer la problématique des erreurs en situation scolaire et de considérer le lexique comme un descripteur de différents phénomènes langagiers et opérations scripturales.

Si l'on s'accorde à reconnaître que la visée intentionnelle ${ }^{4}$ constitue avec l'objet du discours le niveau de structuration le plus élevé et fonde le principe de sélection lexicale le plus puissant, on admettra alors que le lexique investit à ce rang supérieur une représentation sémantique globale qui articule des paramètres énonciatifs (l'ancrage situationnel et l'acte de discours principal) et thématiques (l'objet du discours déployé en une isotopie singulière et dominante).

L'application qui fonde les développements qui viennent est fondée sur le corpus restreint des huit textes déjà évoqués, écrits dans le cadre d'un enseignement en français langue seconde, destiné à des étudiants chinois qui ont opté pour toutes les filières, scientifiques ou littéraires, d'une université française pluridisciplinaire. Les textes écrits sont produits, en temps limité, au mois de décembre. Ils répondent à la consigne suivante :

Des amis vous ont écrit car ils souhaitent organiser une fête du Nouvel An chinois. Ils savent que vous en avez déjà organisé une et vous demandent conseil pour que leur fête soit réussie. Rédigez la lettre de réponse que vous leur faites.

Quel que soit le niveau de compétence atteint par chaque scripteur, on conviendra que le lexique français auquel il doit puiser pour satisfaire le travail d'écriture demandé est thématiquement contraint. Les ressources lexicales sont globalement dénominatives, référentielles, concrètes et nominales : la fête, les invitations, la salle, les plats, etc. Outre cette prévisibilité lexicale qui distribue les sous-thèmes informatifs, le genre épistolaire et l'acte de discours dominant - le conseil d'action programmatique sont également constitutifs de la cohérence générale du discours écrit. Le stock lexical défini actualisé, ordonné et mis en œuvre par un genre communicationnel identifié « configure » la cohérence du texte.

Les erreurs relevées dans les textes étudiés sont présentées ci-dessous dans un ordre qui simule l'ordre de composition des textes et les représentations sémantiques associées : de l'intention signifiante globale (le contexte au sens large) à la réalisation locale d'une forme lexicale, en passant par l'environnement syntaxique d'une unité. Dans la mesure où les scripteurs sont des adultes qui ont déjà une bonne maîtrise conceptuelle, culturelle et linguistique de l'objet de discours à construire, dans leur langue maternelle, on peut penser que cet ordre obéit à une certaine logique. En tout cas, au plan méthodologique, l'ordre retenu livre un préclassement qui permet d'identifier d'emblée les unités lexicales à un statut fonctionnel : il leur affecte une incidence propre, spécifiant un rang de structuration et une modalité descriptive appropriée (pragmatique, syntaxe, sémantique) ${ }^{5}$.

\subsection{Genre d'écrit et contexte au sens large : mise en mots d'une intention signifiante globale (sens intenté) et disponibilité lexicale}

Le sens commun, qu'il s'agisse de répondre à une demande de définition ou d'interroger la pertinence d'un mot choisi, souligne le rôle décisif du contexte d'emploi, ainsi qu'en témoignent les expressions suivantes : "ça dépend, dans quel contexte ? ", «ça ne va pas dans ce contexte-là », " comment ça se dit quand... ». De même, le relevé de certaines « perles » dans les copies d'élèves est souvent fondé sur des écarts (anachronisme, familiarité) sensibles entre le contexte et le vocable utilisé. C'est ainsi qu'on refusera par exemple de considérer, comme un scripteur adolescent nous y invite, que Chateaubriand part faire du camping en Bretagne. Pas plus qu'on ne tolèrera de voir caractériser le massacre de la SaintBathélémy comme une scène à fort taux d'hémoglobine. Certes, mais le jugement normatif ne peut pas en rester à une simple prescription. Il faut approfondir l'intuition commune qui fait du contexte un facteur 
déterminant dans la sélection lexicale et établir les liens de solidarité sémantique et pragmatique qui se jouent entre le sens intenté, la grammaire du discours produit et les faits lexicaux les plus remarquables.

Afin de ne pas augmenter le volume de notre contribution, nous avons consigné ci-dessous des fragments qui nous ont paru problématiques (en italique). Le segment central, identifiant l'erreur, figure en droits. Ensuite, entre crochets, nous proposons une réécriture du segment incriminé et nous caractérisons l'erreur en termes sémantiques et énonciatifs. Autant que faire se peut, nous avons regroupé les problèmes par famille de questions (planification, connecteurs, domaine référentiel, orientation). Notre objectif est ici de guider l'interprétation des textes et d'y traiter les indicateurs lexicaux. Par exemple, l'entrée domaine référentiel identifié (isotopie), associée à l'entrée orientation et point de vue du procès - en l'occurrence, la préparation d'une fête - non seulement favorise l'analyse du traitement lexical de la thématique, mais incite à s'enquérir de toutes les occurrences qui actualisent l'emploi de préparer. Une telle investigation nous conduit à constater l'absence tout à fait remarquable du vocable préparatifs, au profit du verbe préparer et du nom préparation qui lui sont systématiquement préférés. Cette absence, dans ce contexte, endosse bien évidemment le statut d'« erreur lexicale».

Planification, énonciation et modalités : les interactions d'ouverture (salutations et réponse à une requête : demander, donner des conseils) et de clôture épistolaire (formuler des voux de réussite)

- Je suis contente de recevoir ta lettre. Pour organiser la fête de Nouvel An chinois, je te donne des conseils. $<$ J'ai été contente... Je vais te donner quelques conseils $>$. Valeur modale du présent : sémantique et pragmatique des temps verbaux.

- J'ai déjà reçu votre lettre. Tu m'as demandé comment organiser une fête du Nouvel An chinois. Est-ce que tu vas organiser une fête? <Tu vas vraiment organiser une fête ?>. Modalité de l'interrogation (demande de confirmation).

- Salut! Vous allez bien? Voulez-vous organiser une fête du Nouvel An chinois ? C'est vrai ? Ça tombe bien. Je l'ai faite avec mes camarades, il y a deux semaines, et je peux vous donner quelques conseils. < Vous organisez une fête du Nouvel An chinois, vraiment?>. Modalité interrogative: l'inversion du sujet n'est pas compatible, du point de vue de la modalité énonciative, avec la demande de confirmation.

- $\quad$ Il semble que vous allez organiser (...) <Apparemment, vous allez organiser $(\ldots)>$.

- Enfin, je vous souhaite que vous fassiez une bonne fête et bonne année. $<\mathrm{Je}$ vous souhaite de réussir votre fête>. Valeur sémantique et distribution des pronoms personnels en français: gestion de la coréférence (vous). L'atelage sous le coordonnant $e t$, dont la fonction structurelle se rapproche de celle d'un connecteur interphrastique $<$ Et bonnes fêtes !>.

Connecteurs et marqueurs de portée :

- Premièrement c'est l'invitation. <En premier (lieu), il y a l'invitation / Le plus important, ce que vous devez faire en premier, c'est l'invitation>.

- À mon côté $<$ De mon point de vue / À mes yeux>

Domaine référentiel identifié (isotopie) : nous avons cherché dans les textes produits jusqu'à quel point le discours spécifie culturellement (Chine) la fête (fête de Nouvel An) organisée. Nous avons constaté que les textes dans leur majorité traitaient «l'organisation d'une fête », sans aller plus loin dans la spécificité. Cette réduction peut s'interpréter de deux façons : d'une part, les expressions référentielles complexes se prêtent, en seconde mention, à l'effacement du groupe adjoint de détermination; d'autre part, la situation scolaire de production contribue à «dés-authentifier » la lettre de conseils. On peut dès lors comprendre que le locuteur choisisse une voie qu'on qualifiera de moyenne, de standard : il décrit une fête telle qu'il en existe dans tous les pays. Là encore, c'est l'absence de certains vocables (chinois, traditionnel, noms des plats, vocabulaire non traduit, description des costumes, etc.) qui induit à nos yeux l'hypothèse d'un discours scolaire. 
- Une / votre / la fête que vous préparez <La fête du Nouvel An chinois $>$

Orientation et point de vue du procès : préparer et $\left(s^{\prime}\right)$ organiser sont les deux lexèmes de procès les plus fréquents. Étant donné leur rôle supra-processuel, ce n'est pas étonnant; ils recouvrent des verbes à la fois plus spécifiques et moins fréquents tels que décorer, ranger, nettoyer, ou bien ils servent de verbes supports dans des expressions telles que préparer la salle, les plats, etc.

- D'abord il est important que vous prépariez de la fête. Il y a beaucoup de préparations. $<$ D'abord vous devez vous occuper des préparatifs (s'occuper de) $>$. Interférence des deux constructions : s'occuper de et préparer.

- Enfin, pendant la fête, servez des gens invités le meilleur que vous pouvez faire. $<$ Enfin, pendant la fête, servez vos invités (du mieux que vous pouvez) / le mieux possible>. Phraséologie.

Le rapide échantillon d'erreurs que l'on vient de parcourir n'a que peu de rapport avec le lexique à proprement parler, ou tout au moins avec le sens dénoté en langue d'unités lexicales prises hors contexte. Les deux domaines principaux, caractérisant les phénomènes de contexte énonciatif, sont effectivement surtout ceux de la sémantique et de la pragmatique. Nous maintenons cependant cette première catégorie, parce qu'elle sollicite le lexique sur un mode prévisionnel et que, par ailleurs, elle rappelle que les acquisitions lexicales comportent tout un versant phraséologique qui est minoré par la tradition lexicologique française. Enfin, la famille des connecteurs pragmatiques justifierait à elle seule qu'on maintienne les liens entre une entrée pragmatique et des occurrences lexicales.

\subsection{Lexique-grammaire et contexte au sens étroit (le cotexte)}

Dans cette section, nous avons regroupé par familles des problèmes qui relèvent des relations entre le vocable (erroné) et son environnement immédiat. Plusieurs cas se présentent : le cotexte est dominé par une certaine configuration syntaxique quand l'unité fautive implique une transformation d'énoncé attestée (le passif par exemple); le cotexte est figé quand l'unité est complexe et ne peut pas être librement soumise à n'importe quelle transformation (expression, polylexie, phraséologie, gallicisme, etc.). Les domaines d'infraction combinent la représentation sémantique et l'organisation syntaxique.

Quantification et indéfinis : tous les gens, tout le monde, des gens invités, chacun

- (L'organisation d'une fête) ce n'est pas difficile si vous pensez à quelques choses. < Ce n'est pas difficile si vous pensez à deux, trois choses importantes $>$. La marque du pluriel appliquée à quelque chose ne produit pas mécaniquement le même effet que celui qu'on aurait sur quelqu'un I quelques-uns.

- Le nombre de gens qui vont participer < Le nombre d'invités / participants (à la soirée)>.

- Le total de gens $<$ Le nombre de personnes $>$.

Procès, orientation et forme d'énoncé : il est / elle est / c'est. La difficulté de statuer sur il, personnel ou impersonnel, redouble quand la concurrence avec ce, perçu comme plus faiblement référentiel sous la forme élidée $c$ '.

Rôles, voix et thèmes :

- Je souhaite qu'il soit une fête réussie. $<$ Je souhaite que votre fête soit réussie>.

- C'est important que tous les plats soient acceptés par tous. $<$ C'est important que les plats plaisent à tous / soient au goût de tous>. Le passif, associé à «l'objet » du procès.

Évaluatifs : bon, bien, beau

- Est-ce que tu vas organiser une fête ? c'est très bon. <C'est très bien>.

Collocation, expression figée, gallicisme : 
- Voilà. J'espère que vous ferez une bonne fête. <J'espère que votre fête sera réussie>. Le défigement de l'expression bonne fête (faire) et le changement de sens afférent (\# souhaiter la fête de quelqu'un, je vous souhaite une bonne fête).

- Je vous souhaite une fantastique fête. <Je souhaite que votre fête soit très réussie / Je vous souhaite une fête très réussie / Je vous souhaite une fête fantastique>. L'antéposition et le choix de l'adjectif (fantastique) font apparaître une valeur aspectuelle d'accompli (la fête a été fantastique) qui n'est pas recevable dans le contexte (je vous souhaite).

L'échantillon d'erreurs en contexte étroit, ainsi que le classement auquel nous avons soumis les occurrences, atteste de la faible homogénéité linguistique des problèmes rencontrés. La sémantique des quantifieurs, la syntaxe des adjectifs et le recensement lexical d'une famille thématique donnée (les formules de souhaits ou d'évaluatifs), pour déstabilisants qu'ils puissent momentanément paraitre, valident au moins notre hypothèse de faire du lexique un indicateur fonctionnel de l'interface forme-sens.

\subsection{Choix des unités lexicales : organisation et formation du lexique}

À ce troisième niveau, nous trouvons des erreurs que l'on espère pouvoir dire «exclusivement» lexicales. En effet, le contexte devient beaucoup moins utile pour comprendre l'erreur relevée et la « correction» qui opère selon une commutation stricte ne devrait pas faire l'objet d'une hésitation ou d'une discussion normative. Autrement dit, d'un expert à l'autre, il y aura consensus sur le segment à corriger, sur la solution disponible et probablement sur l'explication de l'erreur sanctionnée. C'est à ce stade le versant morphosémantique de la langue qui est essentiellement impliqué (dérivation, construction, composition, figures de mots, relations lexicales).

Construction interne :

- Promoter. $<$ Promouvoir $>$.

- Décorater la place par les décorations. $<$ Décorer (...) avec (...)>.

Construction externe : sélection de la préposition

- Réfléchir les spectacles $<$ Réfléchir à, aux spectacles>.

Partie / tout : choses, mobilier, équipement, matériel, décoration

- D'abord il faut bien équiper. Des colles et des ciseaux sont des outils obligatoires pour décorer la salle. $<\mathrm{N}$ 'oubliez pas de vous équiper en petit matériel ; par exemple pensez à la colle et aux ciseaux dont vous aurez besoin pour décorer la salle>.

- Décorater la place par les décorations. $<$ Décorer (...) avec des guirlandes, des images et des messages de bienvenue que vous aurez rédigés en plusieurs langues>.

- Les outils qu'on va utiliser pour les performances $<$ Le matériel (les ustentiles) dont vous aurez besoin pour les spectacles>.

Synonymie, généricité, polysémie et valeur aspectuelle : endroit, lieu, place (emplacement), salle.

- L'élément le plus important, sans lequel votre fête est destinée à l'échec est l'endroit où vous ferez la fête $<$ Ce qui est capital c'est le choix d'un lieu spacieux et agréable $>$.

- Chacun fait le travail propre $<$ Chacun se consacre à son propre travail $>$.

- Chacun obéit à la disposition du responsable <Chacun se met à la disposition du / obéit au responsable>. Interférence de deux expressions synonymiques qui aboutit à une forme pléonastique inadéquate. 
Dans cette dernière famille d'erreurs, l'unité repérée comme déviante est à part entière une forme-sens relevant d'une description morphosémantique. Les relations entre deux unités éventuellement confondues sont en principe disponibles en lexicologie et en rhétorique pour les emplois dits " figurés ». Quoi qu'il en soit, c'est le domaine où les erreurs lexicales sont marquées.

\section{Bilan provisoire : statut du lexique, aides lexicales, objets d'enseignement et limites d'une typologie}

Notre propos, tout spécialement dans l'application partielle que nous avons exposée sous la forme d'un premier classement d'erreurs, nous a confortées dans l'intuition que le lexique ne répondait pas facilement à un travail classificatoire homogène. Plusieurs arguments nous paraissent pouvoir expliquer les limites d'une telle tentative. Tout d'abord, nous avons vérifié dans un corpus pourtant restreint que l'absence d'unité lexicale est aussi essentielle qu'une unité fautive. Difficile dans ces conditions de

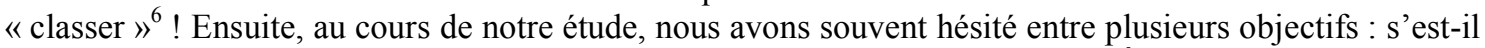
agi de repérer, identifier, décrire, analyser ou expliquer une erreur lexicale ? À vrai dire, notre travail, assez logiquement, a évolué : du repérage initial, nous sommes parvenues à un objectif d'analyse plus ambitieux qui tiendrait compte de la langue comme système et du discours comme instance acutalisante. Enfin et surtout, il nous est apparu que l'unité lexicale, associant une forme à un sens, n'était pas dissociable des données sémantiques et syntaxiques. Par conséquent, le classement esquissé doit être considéré comme un analyseur de ces interrelations et du caractère composite, incomplètement réglé du lexique d'une langue. Cette dimension est cruciale.

Étant donné l'âge et la culture des étudiants chinois dont nous avons étudié les productions, étant donné également l'objet de discours qui leur a été soumis, on peut penser que la représentation conceptuelle et les étapes sémantiques de traitement sont à un stade d'élaboration et de complexité déjà avancé. Cette hypothèse est corroborée par la nature des indicateurs de planification (les connecteurs paradigmatisants du type premièrement, d'abord, etc.). À cet égard, les dichotomies auxquelles recourent les psychologues pour caractériser les phases de la formation du lexique chez un enfant (rigide / souple, simple / complexe, global / analytique, concret / abstrait, subjectif / objectif, instable / stable ; Bramaud du Boucheron 1981 : 82) ne sont pas toutes également efficientes. La capacité à décomposer et analyser un objet conceptuel donné est bien maîtrisée par nos scripteurs, mieux qu'elle ne le serait chez de jeunes élèves. L'observation a deux conséquences. D'une part, il faut de toute évidence renouveler les observations et les élargir à des productions d'élèves plus jeunes, qu'ils soient francophones ou non; les élargir aussi à un public non francophone chez qui l'acquisition du français se situe à un seuil inférieur. D'autre part, concernant les productions des étudiants, il faut poursuivre l'examen minutieux du lexique à l'interface de la syntaxe, de la sémantique et de la pragmatique.

Nous sommes ainsi confortées dans la nécessité de dépasser, en contexte didactique, la séparation trop tranchée entre langue et discours. Les plans d'énonciation, l'aspect, la référence, la quantification, les processus rédactionnels ${ }^{7}$ sont autant de structurations qui pèsent sur la sélection lexicale. Certes, les morphèmes commandés par les composantes sémantique et pragmatique sont traditionnellement appelés des morphèmes grammaticaux ; mais pour utile qu'elle soit, la dichotomie lexique / grammaire mérite au moins un réexamen scrupuleux qui tienne un meilleur compte des propriétés sémantiques.

Que dire enfin de la délicate question des registres et des genres ? Le jugement de disqualification, retenu contre certains énoncés, vaut semble-t-il pour la familiarité qui n'est pas, elle non plus, seulement lexicale. Faute d'avoir approfondi cet aspect, on se contentera de rappeler que la norme d'une homogénéité lexicale est un idéal tout théorique et que la variété des registres à l'intérieur d'un même discours constitue plutôt la norme. Cependant, se contenter d'une telle position serait oublier un peu vite que les exigences d'homogénéité augmentent avec la complexité des objets de discours et le caractère institutionnel des interactions. Ce serait oublier également le rôle de l'école dans la promotion du discours écrit bien formé et dans la diffusion des normes langagières. Il n'est donc pas tenable de se contenter d'un 
discours permissif et incantatoire sur la polyphonie des discours. Il apparait plus prudent de poursuivre et renouveler - les protocoles d'enquête sur les besoins lexicaux tels qu'ils se manifestent en contexte d'enseignement et d'apprentissage, et d'engager une réflexion prudente sur l'enseignement de la langue, et plus particulièrement du lexique.

\section{Références bibliographiques}

[Reichler-]Béguelin, M.-J. (1992). L'approche des anomalies argumentatives. Pratiques, 73, 51-78.

Apothéloz, D. (2002). La construction du lexique français. Paris : Ophrys.

Besse, H., Porquier, R. (1991). Grammaires et didactique des langues. Paris : Hatier / Didier.

Blumenthal, P. (2002). Le centrage du verbe transitif. Syntaxe et sémantique, 4, 15-46.

Bogaards, P. (1994). Le vocabulaire dans l'apprentissage des langues étrangères. Paris : Les Editions Didier.

Bourquin, J. (1980). La dérivation suffixale (théorisation et enseignement) au XIXe siècle. Lille-Paris : diffusion Librairie Honoré Champion.

Bramaud du Boucheron, G. (1981). La mémoire sémantique de l'enfant. Paris : PUF.

Bréal, M. [1921]. (1982). Essai de sémantique. Brionne : Gérard Monfort.

Catach, N. (1979). Le graphème. Pratiques, 25, 21-42.

Catach, N. (1980). L'orthographe française. Paris : Nathan.

Catach, N., Duprez, D., Legris, M. (1980). L'enseignement de l'orthographe. Paris : Nathan, Dossiers didactiques.

Chemlal, S. (2002). Concepts et lexique en développement : une influence réciproque ? Catégorisation et langage, F. Cordier et J. François, dir., Hermès-Lavoisier, 165-181.

Clark, E.-V. (1998). Lexique et syntaxe dans l'acquisition du français. Langue française, 118, 49-60.

Clark, E.-V. (1999). The lexicon in acquisition. Cambridge : Cambridge Studies in linguistics.

Cogis, D. (2005). Pour enseigner et apprendre l'orthographe. Paris : Delagrave.

Corder, P. S. (1980). Que signifient les erreurs des apprenants? Langages, 57, 9-15.

Cordier, F., François, J., dir., Catégorisation et langage. Paris : Hermès-Lavoisier.

Doca, G. (1981). Analyse psycholinguistique des erreurs faites lors de l'apprentissage d'une langue étrangère. Paris : C.I.R.E.R., Publications de la Sorbonne.

Favre, D. (1995). Conception de l'erreur et rupture épistémologique. Revue Française de Pédagogie, 111, 85-94.

Fayol, M. (1997). Des idées au texte. Psychologie cognitive de la production verbale, orale et écrite. Paris : PUF.

Flaux, N., Glatigny, M., Samain, D., éds, (1996). Les noms abstraits. Histoire et théories. Lille : Septentrion.

François, J. (2006). Types de prédications et statut participatif et référentiel de l'objet : l'approche de la ROLE AND REFERENCE GRAMMAR. F. Corblin, S. Ferrando et L. Kupferman, éds, Indéfini et prédication, 293-307.

Frei, H. [1929], (1993). Grammaire des fautes. Genève-Paris : Slatkine Reprints.

Garcia-Debanc, C. (1984). Une évaluation formative en pédagogie de l'écriture. Pratiques, 44, 21-52.

Garcia-Debanc, C. (1990). L'élève et la production d'écrits. Metz : Didactique des textes.

Gey, M. (1979). Que fais-tu en orthographe ? Pratiques, 25, 43-52.

Givon, T. (2001). Syntax : an introduction. Amsterdam/Philadelphie : John Benjamins Publishing Company.

Goldberg, A. E. (1995). A construction grammar approach to argument structure. Chicago: The Univerisity of Chicago Press. 
Grimshaw, J. (2005). Words and structure. Stanford : CSLI.

Gross, G. (1996). Les expressions figées en français. Paris : Ophrys.

Groupe EVA (1991). Évaluer les écrits à l'école primaire. Paris : Hachette.

Gruaz, C. (1979). Thèses HESO pour une rénovation. Pratiques, 25, 12-20.

Guibert, L. (1975). La créativité lexicale. Paris : Larousse.

Guimier, C., éd. (1997). Co-texte et calcul du sens. Caen : Presses Universitaires de Caen.

Halté, J.-F. (1984). L'annotation des copies, variété ou base du dialogue pédagogique. Pratiques, 44, 61-69.

Honvault, R. (1985). Que faire en orthographe au Lycée d'Enseignement Professionnel ? Pratiques, 46, 24-34.

Kerbrat-Orecchioni, C., dir., (1991). La question. Lyon : PUL.

Kleiber, G. (1999). Problèmes de sémantique. La polysémie en question. Lille : Septentrion.

Kleiber, G., Schnedecker, C., Thiessen, A., éds. (2006). La relation PARTIE - TOUT. Louvain-Paris : Editions Peeters.

Klein, W. (1989). L'acquisition de langue étrangère. Paris : Armand Colin.

Le Ny, J.-F. (1979). La sémantique psychologique. Paris : PUF.

Léard, J.-M. (1992). Les gallicismes. Étude syntaxique et sémantique. Paris-Louvain : Duculot.

Les Langues Modernes, 5, (1987). Les erreurs des élèves, qu'en faire? Ou le triomphe de la raison sur la passion.

Lyons, J. (1970). Linguistique générale. Paris : Larousse.

Masseron, C. (2001). Du projet de discours à la langue du discours produit : nature et enjeux des erreurs scripturales, Pratiques, 109-110, 207-247.

Masseron, C. (2005). Indicateurs langagiers et stratégies scripturales - du discours à la langue, Pratiques, 125-126, 205-249.

Monte, M., Touchard, Y., Santacroce, M., Véronique, D., Vion, R. (1992). Quand des enseignants réécrivent des textes d'élèves... Pratiques, 73, 96-118.

Nyckees, V. (1998). La sémantique. Paris : Belin.

Perdue, C. (1980). L'analyse des erreurs : un bilan pratique. Langages, 57, 87-94.

Picoche, J. (1986). Structures sémantiques du lexique français. Paris : Nathan.

Porquier, R. (1977). L'analyse des erreurs. Problèmes et perspectives. Etudes de Linguistique Appliquée, 25, $23-43$.

Porquier, R. (1990). Approche cognitive et didactique des langues: des processus aux exercices. D. Gaonac'h, éd., Acquisition et utilisation d'une langue étrangère. L'approche cognitive. Paris : Hachette. Le Français dans le Monde: recherches et applications.

Porquier, R., Frauenfelder, U. (1980). Enseignants et apprenants face à l'erreur, ou l'autre côté du miroir. Le français dans le monde, 154, 29-36.

Rastier, F. (1987). Sémantique interprétative. Paris : PUF.

Rastier, F. (1989). Sens et textualité. Paris : Hachette.

Rastier, F., Bouquet, S., éds, (2002). Une introduction aux sciences de la culture. Paris : PUF.

Reuter, Y. (1984). Pour une autre pratique de l'erreur. Pratiques, 44, 117-126.

Rey, A. (1977). Le lexique : images et modèles. Du dictionnaire à la lexicologie. Paris : Armand Colin.

Robert, S. (2002). Modèles linguistiques de production. Production du langage, M. Fayol, dir., Paris : Hermès, 6787.

Rossari, C., Beaulieu-Masson, A., Cojocariu, C., Razgouliaeva, A., dir., (2005). Les états de la question. Laval : Nota Bene. 
Talmy, L. (2001). Toward a cognitive semantics. Vol. II : Typology and process in concept structuring. Cambridge, Massachusetts : MIT.

Tarin, R. (2006). Apprentissage, diversité culturelle et didactique. Français langue maternelle, langue seconde ou étrangère. Tournai : Editions LABOR.

Touratier, C. (2005). La sémantique. Paris : Armand Colin.

Tréville, M.-C., Duquette L. (1996). Enseigner le vocabulaire en classe de langue. Paris : Hachette FLE.

Van Valin Jr., R.D., La Polla, R.J. (1997). Syntax: structure, meaning and function. Cambridge: Cambridge University Press.

Veltcheff, C., Hilton S. (2003). L'évaluation en FLE. Paris : Hachette FLE.

Véronique, D. (2000). Recherches sur l'apprentissage des langues étrangères : friches et chantiers en didactique des langues étrangères. Etudes de Linguistique Appliquée, 120, 405-417.

Vigner, G. (2001). Enseigner le français comme langue seconde. Paris : CLE International.

Vogel, K. (1995). L'interlangue - la langue de l'apprenant. Toulouse : Presses universitaires du Mirail.

${ }^{1}$ L'évaluation des capacités en lecture n'a pas été absente du débat sur ces démarches qui visaient à renouveler qualitativement l'analyse des erreurs produites et ainsi à formuler des objectifs d'enseignement sur la base de besoins langagiers mieux identifiés.

${ }^{2}$ De même, l'opposition entre l'oral et l'écrit constitue-t-elle une spécificité - non commune - à prendre en compte dans l'analyse ; plus précisément, la ligne de partage entre le code oral et le code écrit ne joue pas du tout le même rôle dans les deux domaines de l'orthographe et du lexique. Le code oral est constitutif des erreurs et de leurs explications en orthographe, tandis qu'un genre de l'oral identifie un domaine d'application à part entière dans le cas des erreurs lexicales et il statue sur l'existence ou non d'une erreur.

${ }^{3}$ On aura remarqué également que dans l'extrait cité, le terme vote, à la place duquel conviendrait mieux voix, n'est pas « corrigé » par le maître.

${ }^{4}$ Nous incluons, dans la notion d'intention, les données (âge, sexe, culture, niveau scolaire) qui définissent l'identité langagière du locuteur et qui permettent d'induire un seuil plausible de capacités lexicales.

${ }^{5}$ La perspective des erreurs permet peut-être de reconsidérer la part de la langue et des analyses linguistiques - y compris pragmatiques - dans un cadre d'enseignement du français langue étrangère.

${ }^{6}$ On sait qu'en orthographe, l'erreur peut être repérée tout en réalisant un cas de graphème non marqué (les *enfant).

${ }^{7}$ Par exemple, le choix d'une expression résomptive, la solution d'une périphrase définitoire ou d'une valeur figurée, entrerait dans le phénomène cité. 